\title{
Decentralized Coordinated Voltage Control for VSC-HVDC Connected Wind Farms Based on ADMM
}

\author{
Guo, Yifei; Gao, Houlei; Xing, Hao; Wu, Qiuwei; Lin, Zhongwei
}

Published in:

IEEE Transactions on Sustainable Energy

Link to article, DOI:

10.1109/TSTE.2018.2848467

Publication date:

2018

Document Version

Peer reviewed version

Link back to DTU Orbit

Citation (APA):

Guo, Y., Gao, H., Xing, H., Wu, Q., \& Lin, Z. (2018). Decentralized Coordinated Voltage Control for VSC-HVDC Connected Wind Farms Based on ADMM. IEEE Transactions on Sustainable Energy, 10(2), 800 - 810. https://doi.org/10.1109/TSTE.2018.2848467

\section{General rights}

Copyright and moral rights for the publications made accessible in the public portal are retained by the authors and/or other copyright owners and it is a condition of accessing publications that users recognise and abide by the legal requirements associated with these rights.

- Users may download and print one copy of any publication from the public portal for the purpose of private study or research.

- You may not further distribute the material or use it for any profit-making activity or commercial gain

- You may freely distribute the URL identifying the publication in the public portal 


\title{
Decentralized Coordinated Voltage Control for VSC-HVdc Connected Wind Farms Based on ADMM
}

\author{
Yifei Guo, Houlei Gao, Member, IEEE, Hao Xing, Member, IEEE, \\ Qiuwei Wu, Senior Member, IEEE, Zhongwei Lin
}

\begin{abstract}
This paper proposes a decentralized coordinated voltage control scheme (DCVCS) for voltage-source-converter high-voltage dc (VSC-HVdc) connected wind farms based on the Model Predictive Control (MPC) which regulates the voltage profile across the wind farm network within the feasible range by optimally coordinating the VSC and wind turbines (WTs). Firstly, the centralized optimal control problem based on the MPC is formulated to minimize voltage deviations and reactive power output fluctuations of WTs. Secondly, the decentralized solution methodology based on Alternating Direction Method of Multipliers (ADMM) with fast convergency is used to solve the MPC problem in a decentralized manner without loss of optimality of the primal problem. A wind farm with 64 WTs was used to validate the effectiveness and superiority of the proposed DCVCS.
\end{abstract}

Index Terms-alternating direction method of multiplier (ADMM), decentralized control, model predictive control (MPC), voltage control, voltage-source-converter-based high voltage direct current (VSC-HVdc), wind farm.

\section{NOMENCLATURE}

A. Variables

$\Delta(\cdot)$
$\frac{(\cdot)_{d},(\cdot)_{q}}{\bar{V}}, \underline{\underline{V}}$
$\theta, \underline{S}$
$V_{C}$
$V_{S}$
$V_{W}$
$i_{c}$
$i_{s}$
$P_{W}, Q_{W}$

Incremental values of variables dq components of voltages or currents Bus voltage and its conjugate Complex power and its conjugate Phase angle of voltage VSC terminal voltage magnitude VSC controlled bus voltage magnitude Terminal voltage magnitude of WTs Current injection to VSC terminal Current injection to VSC controlled bus Active and reactive power of WT

This work was supported in part by the National Key Research and Development Program of China under Grant 2016YFB0900603, in part by the Taishan Scholar Program, and in part by the China Scholarship Council (CSC).

Y. Guo, H. Gao and Q. Wu are with Key Laboratory of Power System Intelligent Dispatch and Control of Ministry of Education, School of Electrical Engineering, Shandong University, Jinan 250061, China (e-mail: yfguo.sdu@gmail.com; houleig@sdu.edu.cn; quiwudtu@163.com).

H. Xing is with Hangzhou Dianzi University, Hangzhou 310018, China (e-mail: Xing.h@hdu.edu.cn).

Z. Lin is with the State Key Laboratory of Alternate Electrical Power System with Renewable Energy Sources, School of Control and Computer Engineering, North China Electric Power University, Beijing 102206, China (e-mail: lzw@ncepu.edu.cn).
$P_{S}, Q_{S}$

$V_{C}^{\min }, V_{C}^{\max }$

$\Delta V_{C}^{\min }, \Delta V_{C}^{\max }$

$Q_{W}^{\min }, Q_{W}^{\max }$

$\Delta Q_{W}^{\min }, \Delta Q_{W}^{\max }$

B. Parameters

$\bar{Y}_{\text {bus }}$

$N$

$R, L$

$C_{f}$

$\omega$

$K_{p}^{\mathrm{inr}}, K_{i}^{\mathrm{inr}}$

$K_{p}^{\text {out }}, K_{i}^{\text {out }}$

$\tau_{\text {inr }}$

$\tau_{W}$

$\tau_{\mathrm{d}}$

$\alpha_{S}, \alpha_{W}, \alpha_{Q}$

$\rho$

$V_{\text {ref }}$

$H_{p}, H_{c}$

$N_{p}, N_{c}$

$T_{c}$

$\epsilon^{\text {pri }}, \epsilon^{\text {dual }}$

$\varepsilon_{\text {inc }}, \varepsilon_{\text {dec }}$

$\mu$

C. Sets

$\begin{array}{ll}\mathcal{A} & \text { Set of local agents } \\ \mathcal{I} & \text { Set of } P Q \text { buses } \\ \mathcal{N}_{\text {bus }} & \text { Set of buses } \\ \mathcal{N}_{W} & \text { Set of WTs } \\ \mathcal{S} & \text { Set of slack bus } \\ \mathcal{X} & \text { Constraint set }\end{array}$

Active and reactive power injections to VSC controlled bus

Minimum and maximum limit of VSC terminal voltage

Minimum and maximum ramp limit of VSC terminal voltage

Minimum and maximum reactive power limit of WTs

Minimum and maximum reactive power ramp limit of WTs

Admittance matrix

Number of buses in offshore wind farm Resistance and inductance of the phase reactor

Effective capacitance of the filter

Angular frequency

PI parameters in the inner loop of VSC

PI parameters in the outer loop of VSC

Time constant of inner loop of VSC

Time constant of reactive power loop of WTs

Time delay

Weighting factors

Penalty parameter

Voltage reference

Prediction and control horizons

Prediction and control steps

Control period

Tolerances for primal and dual residuals

Tunable parameters for $\rho$-updating

Tunable parameters for $\rho$-updating

\section{INTRODUCTION}


$\mathbf{W}$ IND generation has been widely used over the world in past few decades for the growing concerns of energy shortage and global warming [1]. Particularly, a large number of offshore wind farms (OWFs) have been developed due to the advantages of greater marine wind resource, land saving, lower visual and acoustic effects, etc, compared with the onshore ones [2]-[3]. For connecting large-scale remote OWFs, the voltage-source-converter high-voltage dc (VSC$\mathrm{HVdc}$ ) is preferred to other transmission technologies in costbenefit terms when cable lengths exceed 80-120 km [4]-[5].

With the increasing penetration of wind power, modern wind farms are no longer simply regarded as energy resources [6]. They are required not only to meet the specific grid codes set by transmission system operators (TSOs), but also to provide ancillary services such as frequency support, voltage/reactive power support, congestion management as well as damping of electro-mechanical oscillations. Otherwise, wind power might bring great disturbances into power systems due to the stochastic and intermittent nature of wind energy.

The voltage and reactive power management of wind farms, as one of the major topics of regarding wind power integration, has motivated a great number of studies. For conventional acconnected wind farms, several voltage/reactive power control strategies were designed to regulate the voltage at the point of connection (POC) [7]-[8], in which the total required reactive power is calculated based on the voltage at the POC using the slope-based function or using the proportionalintegral (PI) controller and then proportionally dispatched to each wind turbine (WT) according to their available reactive power capability. In recent years, the optimization-based control strategies have been developed to pursue the optimized control performance. In [9], a optimal power flow (OPF)-based reactive power dispatch method was proposed to reduce the electrical losses including not only the losses in the collector cables and transformers but also losses inside WTs. In [10], a sensitivity-based hierarchical optimal voltage control method was designed and implemented in a wind farm in Northern China. In [11], a coordinated voltage strategy based on the Model Predictive Control (MPC) was presented, aiming to coordinate multiple voltage control regulation devices such as static Var compensators (SVCs), on-load tap changing (OLTC) transformer and WTs that have different response time. In [12], a combined active and reactive power control strategy was proposed to regulate the voltage while reducing the fatigue loads of WTs.

With rapid development of offshore wind power projects, voltage/reactive power control of $\mathrm{HVdc}$ connected-OWFs has become an emerging research hotspot in the last few years. Unlike the reactive power/voltage control in conventional ac connected wind farms, which aims to fulfill the TSO voltage requirements at the POC specified by grid codes [13], the aim of OWFs' voltage control is to maintain the terminal voltage of WTs within the feasible range since the VSC-HVdc system decouples the OWFs from the onshore AC grid. Moreover, the wind farm side VSC (WFVSC) is able to provide fast voltage/reactive power regulation capability.

In [14]-[15], the OPF models for reactive power dispatch of HVdc connected OWFs were developed, which optimizes the reactive power outputs of the WTs. As an extension of [15], the voltage control capability of WFVSC was considered as a control variable in [16]. In [17], a bi-level coordinated voltage control scheme was proposed in which the offline OPF method is adopted for the upper level and the proportional-integral (PI) control is used for the lower level. A comparison study of several different control strategies, both conventional and optimization-based control for HVdc-connected OWF clusters, was presented in [18]. In [19], the MPC-based coordinated voltage control strategies were proposed, in which the WFVSC and WTs are optimally coordinated to regulate the network voltage while reducing the system losses. And in [20], an enhanced voltage control scheme for HVdc-connected OWFs was developed which considers the impact of active power on voltage.

As presented above, the previous research has made great contributions to the voltage/reactive power management of wind farms, especially for conventional ac-connected wind farms. However, most of the existing voltage control strategies are designed in a centralized manner such as the OPF-based or sensitivity-based optimization methods. Generally, the wind farm can be regarded as a constrained multiple input and multiple output (MIMO) system whose order dramatically grows with the increasing number of WTs, making the centralized control impractical for the future large-scale OWFs or OWF clusters with hundreds or even thousands of WTs owing to the heavy computation burden of the central controller. The conventional control schemes such as the droop-based or PI control without optimization might fail to obtain the optimal control performance.

This paper proposes a decentralized coordinated voltage control scheme (DCVCS) for VSC-HVdc connected OWFs to regulate voltages within the feasible range by optimally coordinating the WFVSC and WTs. Firstly, the MPC-based voltage control problem of OWFs, aiming to correct voltage deviations as well as smoothen reactive power fluctuations of WTs, is formulated as a multi-step optimization model and transformed into a standard quadratic programming (QP) form. Secondly, a decentralized solution method based on the Alternating Direction Method of Multipliers (ADMM) algorithm is proposed to efficiently solve the problem in a decentralized manner. An analytical sensitivity calculation method [21] is used in this paper to calculate the voltage sensitivity with respect to power injections and slack bus voltage in real-time. The main contributions of this paper can be summarized as follows:

- A decentralized coordinated voltage control scheme is designed. Compared with the centralized control, the proposed DCVCS significantly reduces the computation burden of the central controller without loss of the optimality of the solution, improves the cyber security of the OWFs and potentially has better flexibility and robustness.

- The ADMM algorithm is used to decentralize the wind farm voltage control design, which guarantees the optimality of the control performance.

The rest of this paper is organized as follows. In Section II, 


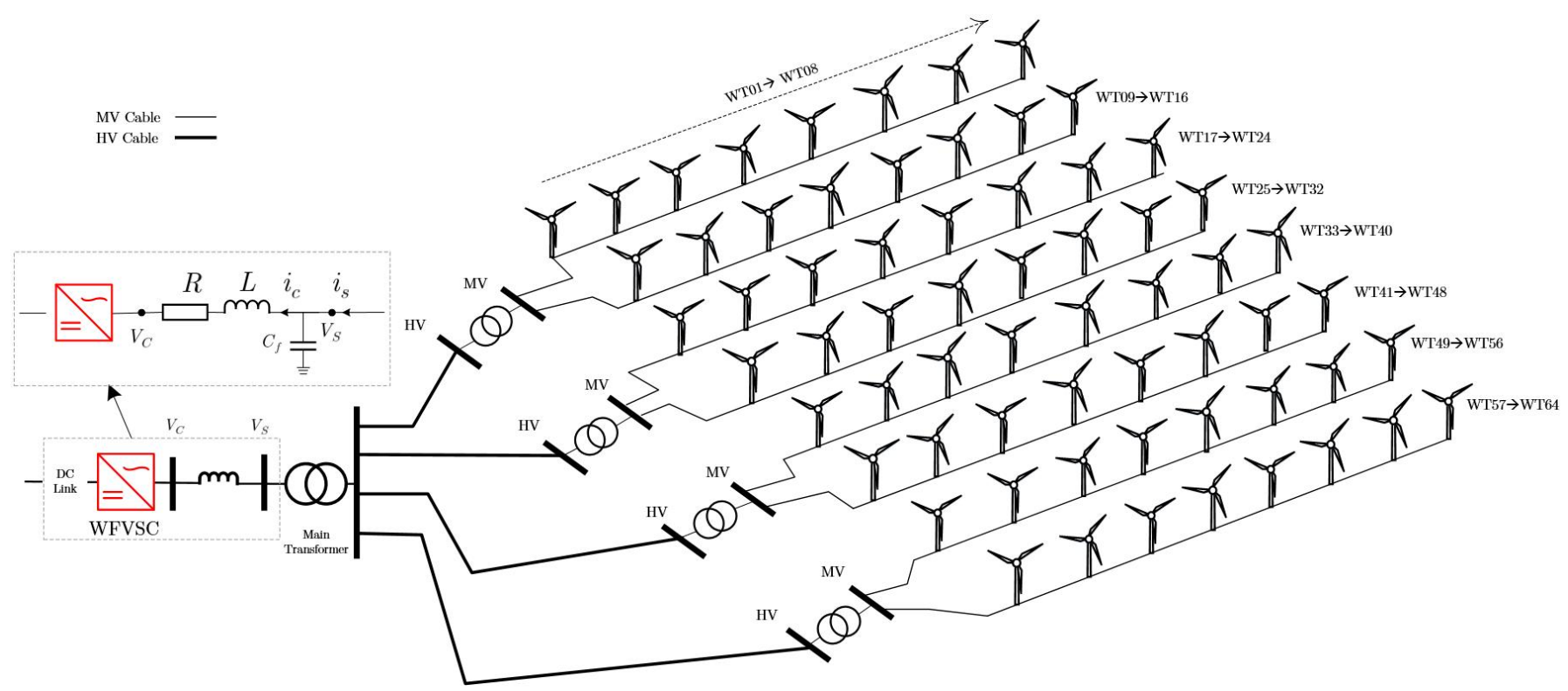

Fig. 1. Structure of VSC-HVDC connected OWFs

a brief overview of the proposed DCVCS is given. In Section III, the analytical sensitivity calculation method is presented. In Section IV, the MPC-based voltage control problem is described. In Section V, the ADMM-based decentralized solution framework is proposed. The simulation results are presented in Section VI. Several potential advantages of the proposed DCVCS compared with the centralized control are discussed in Section VII, followed by conclusions.

\section{OVERVIEW of the DeCEntralized Coordinated Voltage Control SCHEME}

\section{A. Structure of VSC-HVdc Connected Wind Farms}

The typical structure of a VSC-HVdc connected OWF with a common WFVSC is illustrated in Fig. 1. The WTs are collected through MV cables as a string. Every two feeders are equipped with a collector transformer. The power generated by the OWFs is transmitted to the onshore ac grids through a VSC-HVdc transmission system.

\section{B. Structure of the Decentralized Voltage Control}

The structure of the proposed decentralized control is illustrated in Fig. 2. The central agent receives the operation information of the wind farms from the SCADA system. The sensitivity calculation block is used to update the voltage sensitivity coefficients with respect to power injections and slack bus voltage. Here, an analytical calculation method originally developed for radial distribution networks is used to calculate the sensitivity to improve the computational efficiency [21]. The WFVSC and WTs are equipped with a smart agent which can exchange information with the central agent and generate (voltage/reactive power) set-points for WFVSC and WTs.

In this MPC-based voltage control design, the voltage deviations and reactive power fluctuations are taken into account. In the proposed ADMM-based decentralized solution framework,

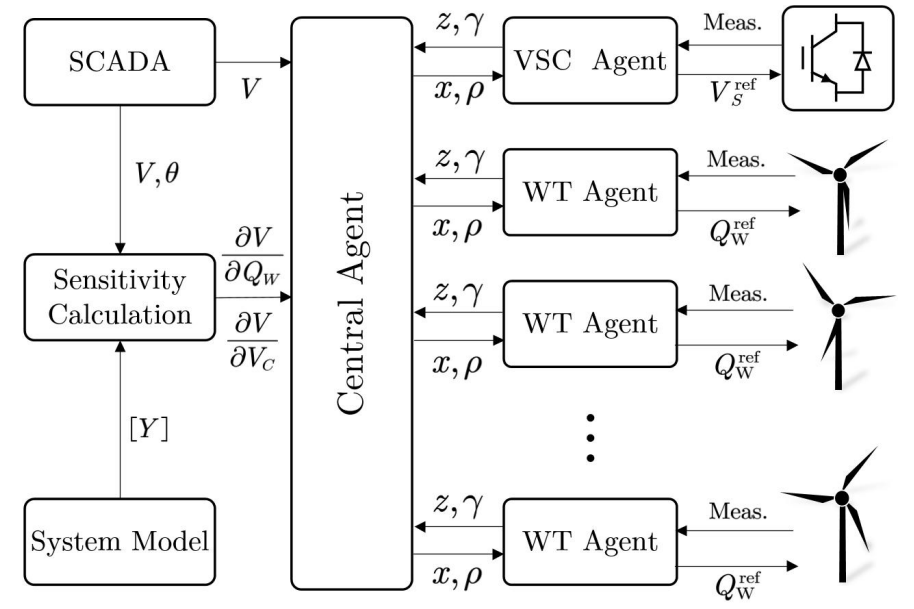

Fig. 2. Structure of the decentralized coordinated voltage control scheme.

the central agent solves an unconstrained optimization problem, largely reducing the computation burden of the central agent. And since the MPC problem is developed based on the convex QP model, the analytical optimal solution can be directly obtained through the simple matrix calculation without any iteration. Each local agent solves a local small-scale constrained optimization problem, which is built based on the local operation information and the iteration information from the central agent.

The proposed control scheme aims to deal with the voltage issues in steady-state operation instead of the transient faultride-through (FRT) operation. For real-life implementation, the coordination between the DCVCS and FRT control schemes of a wind farm should be addressed. Generally, the FRT control should have the higher priority than the steady-state optimal control, i.e., once the FRT control is triggered, the DCVCS should be locked to avoid negative interaction. 


\section{Voltage Sensitivity Calculation}

Suppose the network is comprised of $N$ buses. $\mathcal{S}$ and $\mathcal{I}$ denote the set of slack buses and the buses with $P Q$ injections, respectively (i.e. $\mathcal{S} \cup \mathcal{I}=\mathcal{N}_{\text {bus }}$ and $\mathcal{S} \cap \mathcal{I}=\emptyset$ ). Define the voltage $\bar{V}_{i}:=V_{i} e^{\mathrm{j} \theta_{i}}$ for $i \in \mathcal{S} \cup \mathcal{I}$ and complex power injection $\bar{S}_{i}=P_{i}+\mathrm{j} Q_{i}$ for $i \in \mathcal{I}$. The link between bus voltages and power injections is,

$$
\underline{S}_{i}=\underline{V}_{i} \sum_{j \in \mathcal{S} \cup \mathcal{I}} \bar{Y}_{\text {bus }, i j} \bar{V}_{j}
$$

The following derivations are all based on this equation.

\section{A. Voltage Sensitivity With Respect to Reactive Power Injec-} tions

Firstly, the partial derivatives of $\underline{S}_{i}$ for $i \in \mathcal{I}$ with respect to reactive power injection $Q_{l}$ of a bus $l \in \mathcal{I}$ should be calculated, which satisfy the following equations,

$$
\begin{aligned}
\frac{\partial \underline{S}_{i}}{\partial Q_{l}}=\frac{\partial\left\{P_{i}-\mathrm{j} Q_{i}\right\}}{\partial Q_{l}}=\frac{\partial \underline{V}_{i}}{\partial Q_{l}} \sum_{j \in \mathcal{S} \cup \mathcal{I}} \bar{Y}_{\text {bus }, i j} \bar{V}_{j} \\
+\underline{V}_{i} \sum_{j \in \mathcal{I}} \bar{Y}_{\text {bus }, i j} \frac{\partial \bar{V}_{j}}{\partial Q_{l}}= \begin{cases}-\mathrm{j} 1, & \text { for } i=l . \\
0, & \text { for } i \neq l .\end{cases}
\end{aligned}
$$

It can be observed that (2) is linear with respect to $\partial \bar{V}_{i} / \partial Q_{l}$ and $\partial \underline{V}_{i} / \partial Q_{l}$. According to the theorem in [21], (2) has a unique solution for radial networks (The wind farm has a radial topology). Thus, once $\partial \bar{V}_{i} / \partial Q_{l}$ and $\partial \underline{V}_{i} / \partial Q_{l}$ are obtained, the voltage sensitivity coefficients with respect to power injections can be calculated by,

$$
\frac{\partial V_{i}}{\partial Q_{l}}=\frac{1}{V_{i}} \operatorname{Re}\left(\underline{V}_{i} \frac{\partial \bar{V}_{i}}{\partial Q_{l}}\right) .
$$

\section{B. Voltage Sensitivity With Respect to Slack Bus Voltage}

For a bus $i \in \mathcal{I}$, the partial derivatives with respect to voltage magnitude $V_{k}$ (In this study, only one slack bus exists, namely the terminal bus of WFVSC.) are derived by,

$-\bar{V}_{i} \bar{Y}_{\text {bus }, i k} e^{\mathrm{j} \theta_{k}}=\underline{W}_{i k} \sum_{j \in \mathcal{S} \cup \mathcal{I}} \bar{Y}_{\text {bus }, i j} \bar{V}_{j}+\underline{V}_{i} \sum_{j \in \mathcal{I}} \bar{Y}_{\text {bus }, i j} \bar{W}_{j k}$

where

$$
\bar{W}_{i k}:=\frac{\partial \bar{V}_{i}}{\partial V_{k}}=\left(\frac{1}{V_{i}} \frac{\partial V_{i}}{\partial V_{k}}+\mathrm{j} \frac{\partial \theta_{i}}{V_{k}}\right) \bar{V}_{i} .
$$

Similarly, (4) is linear with respect to $\bar{W}_{i k}$ and $\underline{W}_{i k}$, and it also has a unique solution for a radial network. Then, by solving it, the sensitivity coefficients with respect to the slack bus voltage magnitude at bus $k \in \mathcal{S}$ can be calculated by,

$$
\frac{\partial V_{i}}{\partial V_{k}}=V_{i} \operatorname{Re}\left(\frac{\bar{W}_{i k}}{\bar{V}_{i}}\right)
$$

\section{MPC FORMULATION}

In the MPC, as known, $N_{p} \geq N_{c}$. From the computational viewpoint, they should be equal unless the controller is required to consider changes beyond the control horizon, i.e., $N_{p}=N_{c}$. In this section, the predictive model is given firstly and then the MPC problem formulation is presented.

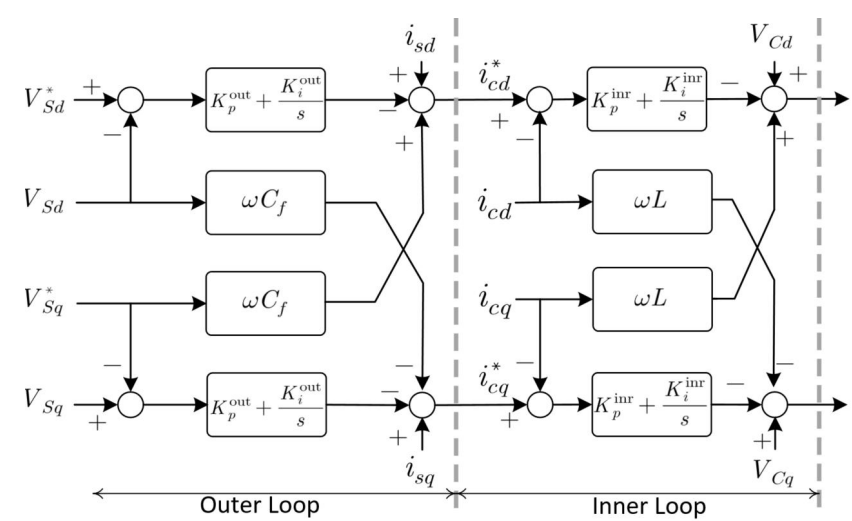

Fig. 3. Control structure of WFVSC.

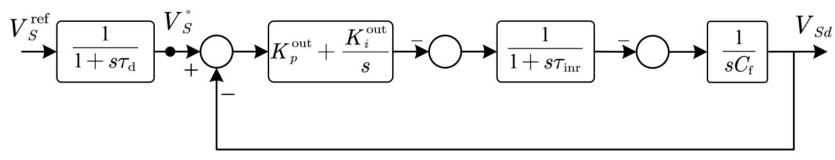

Fig. 4. Equivalent voltage control loop.

\section{A. Predictive Model}

1) Wind Farm Side VSC: The control system of the WFVSC is designed as in Fig. 3 with the typical cascaded outer and inner loops [22]. By introducing the feedforward terms $\omega C_{f} V_{S d}, \omega C_{f} V_{S q}$ for the outer loop and $\omega L i_{c d}, \omega L i_{c q}$ for the inner loop, the $d q$-axis control loops can be decoupled. The feed-forward terms $i_{s d}$ and $i_{s q}$ are obtained based on the measured active and reactive power $i_{s d}=P_{S} /\left(1.5 V_{S d}\right)$ and $i_{s q}=-Q_{S} /\left(1.5 V_{S d}\right)$. By selecting PI parameters $K_{p}^{\mathrm{inr}}=L / \tau_{\mathrm{inr}}$ and $K_{i}^{\mathrm{inr}}=R / \tau_{\mathrm{inr}}$, the inner loop can be modelled as a first-order block with the time constant $\tau_{\text {inr. }}$. Finally, the equivalent voltage control loop including the physical model and external controller is shown in Fig. 4. Accordingly, suppose the current time is $t_{0}$, by defining $\Delta V_{S d}:=V_{S d}-V_{S d}\left(t_{0}\right), \Delta V_{S d}^{*}:=V_{S d}^{*}-V_{S d}\left(t_{0}\right)$, $\Delta i_{c d}:=i_{c d}-i_{c d}\left(t_{0}\right), \Delta V_{S d}^{\text {int }}:=\left(\Delta V_{S d}^{*}-\Delta V_{S d}\right) / s$ and $\Delta V_{S}^{\text {ref }}:=V_{S}^{\text {ref }}-V_{S}\left(t_{0}\right)$, the predictive incremental model of the WFVSC can be expressed as,

$$
\Delta \dot{x}_{V}=\boldsymbol{A}_{V} \Delta x_{V}+\boldsymbol{B}_{V} \Delta u_{V},
$$

with $\quad \Delta x_{V}:=\left[\Delta V_{S d}^{*}, \Delta V_{S d}, \Delta V_{S d}^{\mathrm{int}}, \Delta i_{c d}\right]^{T}, \Delta u_{V}:=$ $\left[\Delta V_{S}^{\mathrm{ref}}\right]$,

$$
\boldsymbol{A}_{V}=\left[\begin{array}{cccc}
-\frac{1}{\tau_{\mathrm{d}}} & 0 & 0 & 0 \\
0 & 0 & 0 & \frac{1}{C_{f}} \\
1 & -1 & 0 & 0 \\
-\frac{K_{p}^{\text {out }}}{\tau_{\text {inr }}} & \frac{K_{p}^{\text {out }}}{\tau_{\text {inr }}} & \frac{K_{i}^{\text {out }}}{\tau_{\text {inr }}} & -\frac{1}{\tau_{\text {inr }}}
\end{array}\right], \boldsymbol{B}_{V}=\left[\begin{array}{c}
\frac{1}{\tau_{\mathrm{d}}} \\
0 \\
0 \\
0
\end{array}\right] .
$$

2) Wind Turbine: Define the $\Delta Q_{W}=Q_{W}-Q_{W}\left(t_{0}\right)$ and $\Delta Q_{W}^{\mathrm{ref}}=Q_{W}^{\mathrm{ref}}-Q_{W}\left(t_{0}\right)$. The dynamic behavior of $Q$-loop of WTs can be modeled as a first-order function [8],

$$
\Delta Q_{W}=\frac{1}{1+s \tau_{W}} \Delta Q_{W}^{\mathrm{ref}}
$$


where $\tau_{W}$ is the time constant which is in the range of $1 \sim 10 \mathrm{~s}$ [23]. $s$ denotes the complex variable. The available reactive power of a FSC-WT can be approximately expressed by [17],

$$
Q_{\mathrm{W}}^{\max }=\sqrt{S_{\mathrm{W}}^{2}-P_{\mathrm{W}}^{2}} .
$$

Accordingly, the predictive incremental state-space model of a WT can be expressed by,

$$
\Delta \dot{x}_{W}=\boldsymbol{A}_{W} \Delta x_{W}+\boldsymbol{B}_{W} \Delta u_{W}
$$

with $\Delta x_{W}:=\left[\Delta Q_{W}\right], \Delta u_{W}:=\left[\Delta Q_{W}^{\mathrm{ref}}\right], \boldsymbol{A}_{W}=\left[-1 / \tau_{W}\right]$, and $\boldsymbol{B}_{W}=\left[1 / \tau_{W}\right]$.

3) System: Firstly, define the incremental state variable vector $\Delta x:=\left[\Delta x_{V}^{T}, \Delta x_{W}^{T}\right]^{T}$, input variable vector $\Delta u:=$ $\left[\Delta u_{V}^{T}, \Delta u_{W}^{T}\right]^{T}$, and the output variable vector $\Delta y:=$ $\left[\Delta V_{C}, \Delta Q_{W_{1}}, \ldots, \Delta Q_{W_{N_{W}}}\right]^{T}$. The incremental voltage of the VSC-controlled bus $\Delta V_{S}$, which is affected by WTs and WFVSC, can be calculated based on the first-order Taylor approximation as,

$$
\begin{aligned}
\Delta V_{S} \approx & \frac{\partial V_{S}}{\partial V_{C}} \Delta V_{C}+\frac{\partial V_{S}}{\partial Q_{W_{1}}} \Delta Q_{W_{1}}+\frac{\partial V_{S}}{\partial Q_{W_{2}}} \Delta Q_{W_{2}} \\
& +\cdots+\frac{\partial V_{S}}{\partial Q_{W_{N_{W}}}} \Delta Q_{W_{N_{W}}}
\end{aligned}
$$

Then, the predictive incremental state-space model of the whole system can be expressed as,

$$
\begin{aligned}
\Delta \dot{x} & =\boldsymbol{A} \Delta x+\boldsymbol{B} \Delta u \\
\Delta y & =\boldsymbol{C} \Delta x
\end{aligned}
$$

with

$$
\begin{aligned}
& \boldsymbol{A}=\left[\begin{array}{c|ccc}
\boldsymbol{A}_{V} & & & \\
\hline & \boldsymbol{A}_{W_{1}} & & \\
& & \ddots & \\
& & & \boldsymbol{A}_{W_{N_{W}}}
\end{array}\right], \\
& \boldsymbol{B}=\left[\begin{array}{c|ccc}
\boldsymbol{B}_{V} & & & \\
\hline & \boldsymbol{B}_{W_{1}} & & \\
& & \ddots & \\
& & & \boldsymbol{B}_{W_{N_{W}}}
\end{array}\right] \\
& \boldsymbol{C}=\left[\begin{array}{l|lll}
\boldsymbol{C}_{V} & \boldsymbol{C}_{W_{1}}^{V} & \cdots & \boldsymbol{C}_{W_{N_{W}}}^{V} \\
\hline & C_{W_{1}} & & \\
& & \ddots & \\
& & & \boldsymbol{C}_{W_{N_{W}}}
\end{array}\right],
\end{aligned}
$$

where

$$
\begin{gathered}
\boldsymbol{C}_{V}=-\left(\frac{\partial V_{S}}{\partial V_{C}}\right)^{-1}[0,-1,0,0], \boldsymbol{C}_{W_{i}}=[1] \\
\boldsymbol{C}_{W_{i}}^{V}=\left[-\left(\frac{\partial V_{S}}{\partial V_{C}}\right)^{-1}\left(\frac{\partial V_{S}}{\partial Q_{W_{i}}}\right)\right], \forall i \in \mathcal{N}_{W} .
\end{gathered}
$$

Then, suppose the sampling period is $\Delta \tau_{s}$, the discrete predictive model of the system can be derived from the continuous model which is,

$$
\begin{aligned}
\Delta x(k+1) & =\boldsymbol{A}_{d} \Delta x(k)+\boldsymbol{B}_{d} \Delta u(k) \\
\Delta y(k) & =\boldsymbol{C}_{d} \Delta x(k)
\end{aligned}
$$

where $\boldsymbol{A}_{d}=e^{\boldsymbol{A} \Delta \tau_{\mathrm{s}}}, \boldsymbol{B}_{d}=\int_{0}^{\Delta \tau_{\mathrm{s}}} e^{\boldsymbol{A} \Delta \tau_{s}} \boldsymbol{B} d \tau, \boldsymbol{C}_{d}=\boldsymbol{C}$.

\section{B. Mathematical Formulation of the Voltage Control Problem}

1) Objective function: The first part of the objective function is explicitly presented as,

$f_{V}=\sum_{k=1}^{N_{p}} \alpha_{S}\left(V_{S}(k)-V_{\mathrm{ref}}\right)^{2}+\sum_{k=1}^{N_{p}} \sum_{i=1}^{N_{W}} \alpha_{W}\left(V_{W_{i}}(k)-V_{\mathrm{ref}}\right)^{2}$.

To be noticed, the unified voltage profile across the wind farm network could be helpful to reduce the power losses of the network as well. The detailed proof can be referred to [24].

The second parts of the control objective is to smoothen the reactive power outputs of WTs, which is described by,

$$
f_{Q}=\sum_{k=1}^{N_{p}} \sum_{i=1}^{N_{W}} \alpha_{Q}\left(Q_{W_{i}}(k)-Q_{W_{i}}(k-1)\right)^{2}
$$

Accordingly, the the total objective function can be expressed by,

$$
f=f_{V}+f_{Q}
$$

2) Constraints: The constraints of WFVSC and WTs at $k$ th step $\left(k=1,2, \ldots, N_{p}\right)$ can be expressed as,

$$
\begin{aligned}
V_{C}^{\min } & \leq V_{C}(k) \leq V_{C}^{\max } \\
\Delta V_{C}^{\min } & \leq V_{C}(k)-V_{C}(k-1) \leq \Delta V_{C}^{\max } \\
Q_{W_{i}}^{\min } & \leq Q_{W_{i}}(k) \leq Q_{W_{i}}^{\max }, \forall i \in \mathcal{N}_{W} \\
\Delta Q_{W_{i}}^{\min } & \leq Q_{W_{i}}(k)-Q_{W_{i}}(k-1) \leq \Delta Q_{W_{i}}^{\max }, \forall i \in \mathcal{N}_{W} .
\end{aligned}
$$

The $k$ th step predicted reactive power outputs of WTs $Q_{W}(k)$ and terminal voltage of WFVSC $V_{C}(k)$ follow the state-space model (12), denoted by the vector $y$. The predicted voltages of $V_{S}(k)$ and $V_{W}(k)$ are calculated based on the firstorder Taylor approximation as,

$$
\begin{aligned}
& V_{S}(k)=V_{S}(0)+\frac{\partial V_{S}}{\partial V_{C}} \Delta V_{C}(k)+\frac{\partial V_{S}}{\partial Q_{W_{1}}} \Delta Q_{W_{1}}(k) \\
& +\frac{\partial V_{S}}{\partial Q_{W_{2}}} \Delta Q_{W_{2}}(k)+\cdots+\frac{\partial V_{S}}{\partial Q_{W_{N_{W}}}} \Delta Q_{W_{N_{W}}}(k)
\end{aligned}
$$

$$
\begin{aligned}
& V_{W_{i}}(k)=V_{W_{i}}(0)+\frac{\partial V_{W_{i}}}{\partial V_{C}} \Delta V_{C}(k)+\frac{\partial V_{W_{i}}}{\partial Q_{W_{1}}} \Delta Q_{W_{1}}(k) \\
& +\frac{\partial V_{W_{i}}}{\partial Q_{W_{2}}} \Delta Q_{W_{2}}(k)+\cdots+\frac{\partial V_{W_{i}}}{\partial Q_{W_{N_{W}}}} \Delta Q_{W_{N_{W}}}(k) .
\end{aligned}
$$

\section{ADMM-BAsed Decentralized Solution FRAMEWORK}

As known, the centralized optimization algorithms might not be suitable for the large systems. To address this issue, in this section, the decentralized solution framework based on the ADMM algorithm [26] is proposed. 


\section{A. Compact QP Form of the MPC Problem}

To clearly derive the ADMM-based decentralized solution, we rearrange the MPC problem into a compact QP form. Hereinafter, the control variable vector is replaced by the decision variable vector $x$ in the optimization problem to clearly present the standard ADMM framework. Accordingly, the MPC problem formulation presented in Section IV can be rewritten into a compact $\mathrm{QP}$ form as,

$$
\begin{array}{ll}
\min _{x} \Phi(x)= & \frac{1}{2} x^{T} \boldsymbol{H} x+\boldsymbol{g}^{T} x \\
\text { s.t. } & x \in \mathcal{X}
\end{array}
$$

where $\boldsymbol{H} \succeq 0$ is the Hessian matrix and $\boldsymbol{g}$ is the coefficient matrix. The constraint set $\mathcal{X}=\mathcal{X}_{1} \times \cdots \times \mathcal{X}_{n}$ where $n=$ $N_{c} \times\left(N_{W}+1\right)$ is the total number of the decision variables.

\section{B. Decentralized Solution Method Based on ADMM}

The basic concept of the ADMM algorithm is to iteratively optimize the augmented Lagrangian over three types of variables: primal variables $x$, auxiliary variables $z$ and dual variables $\gamma$ [26]. We cyclically update one type of variables while fixing other variables, which allows us to solve the problem in a decentralized manner and achieve convergence to the optimal solution obtained by a centralized manner.

Firstly, since $\Phi$ and $\mathcal{X}$ are convex, the problem in (22) can be rewritten into a standard ADMM form as,

$$
\begin{array}{cl}
\min & \Phi(x)+I_{\mathcal{X}}(z) \\
\mathrm{s.t.} & x-z=0 \\
& x \in \mathcal{X}
\end{array}
$$

where $I_{\mathcal{X}}(\cdot)$ is the indicator function of $\mathcal{X}$. The augmented Lagrangian $L_{\rho}$ can be expressed as,

$$
L_{\rho}(x, z, \gamma)=\Phi(x)+I_{\mathcal{X}}(z)+\gamma^{T}(x-z)+\frac{\rho}{2}\|x-z\|_{2}^{2}
$$

In the augmented Lagrangian function $L_{\rho}$, the equality constraints are regularized by an additional quadratic term. More basic knowledge of the ADMM algorithm can be referred to [26].

The ADMM-based DCVCS is solved using the following updating steps:

1) Update $x$ : Theoretically, as known, any bus voltage inside the wind farm network is related to the power injections of all WTs and WFVSC terminal voltage set-point. Thus, the objective function in (22) is coupled with all decision variables and consequently the objective function cannot be directly decomposed. However, the constraints in (16)-(19) are local. Thus, based on the calculation results of $z$ and $\gamma$ at step $k$, obtained from the local agents (WT and WFVSC agent), the primal variables $x$ are updated in the central agent by solving the following unconstrained QP problem,

$$
\begin{aligned}
x^{[k+1]}: & =\underset{x}{\arg \min } L_{\rho}\left(x, z^{[k]}, \gamma^{[k]}\right) \\
& :=\underset{x}{\arg \min }\left\{\Phi(x)+\left(\gamma^{[k]}\right)^{T}\left(x-z^{[k]}\right)\right. \\
& \left.+\frac{\rho^{[k]}}{2}\left\|x-z^{[k]}\right\|_{2}^{2}\right\}
\end{aligned}
$$

since $\boldsymbol{H}+\rho \boldsymbol{I}$ is invertible $(\boldsymbol{H}+\rho \boldsymbol{I} \succ 0)$ where $\boldsymbol{I}$ is the identity matrix., the analytical solution can be obtained by setting $\nabla_{x} L_{\rho}=0$,

$$
x^{[k+1]}=-\left(\boldsymbol{H}+\rho^{[k]} \boldsymbol{I}\right)^{-1}\left(\boldsymbol{g}-\rho^{[k]} z^{[k]}+\gamma^{[k]}\right) .
$$

Consequently, in this step, only the simple matrix calculation is required. Before solving the optimization problem, the received variables $z$ and $\gamma$ from each agent should firstly be sorted consistent with the decision vector $x$ as defined in (22) (In this study, the decision variable is sorted according to the prediction step).

2) Update $z$ : Since the constraints in (16)-(19) are local constraints, in this step, the augmented Lagrangian can be decomposed as,

$$
L_{\rho}\left(x^{[k+1]}, z, \gamma^{[k]}\right)=\sum_{i \in \mathcal{A}} L_{\rho}^{(i)}\left(x_{i}^{[k+1]}, z_{i}, \gamma_{i}^{[k]}\right) .
$$

Therefore, the auxiliary variable $z$ can be updated in each local agent of WFVSC and WTs by minimizing the augmented Lagrangian with respect to $z_{i}$, i.e.,

$$
\begin{aligned}
z_{i}^{[k+1]}:= & \underset{z_{i} \in \mathcal{X}_{i}}{\arg \min }\left\{\left(\gamma_{i}^{[k]}\right)^{T}\left(x_{i}^{[k+1]}-z_{i}\right)\right. \\
& \left.+\frac{\rho^{[k]}}{2}\left\|x_{i}^{[k+1]}-z_{i}\right\|_{2}^{2}\right\}, \quad \forall i \in \mathcal{A} .
\end{aligned}
$$

The small-scale constrained optimization problem with respect to $z_{i}$ can be parallelly and efficiently solved by the local agents. In the first step, we just solve an unconstrained QP problem without considering the constraint on $x$. This modification will not affect the optimality of the primal problem, since the final feasible point satisfies $x^{\star}=z^{\star}$ and $z^{\star} \in \mathcal{X}$.

3) Update $\gamma$ : The dual variable $\gamma_{i}$ can be updated in the central agent or each local agent. In this paper, the dual variables are updated in the local agents as,

$$
\gamma_{i}^{[k+1]}:=\gamma_{i}^{[k]}+\rho^{[k]}\left(x_{i}^{[k+1]}-z_{i}^{[k+1]}\right), \forall i \in \mathcal{A} .
$$

4) Update $\rho$ : To improve the convergence rate in practice, as well as make the convergence performance less dependent on the initial choice of the penalty parameter $\rho$, a varying $\rho$ is applied in this paper, which is updated by,

$$
\rho^{[k+1]}:= \begin{cases}\varepsilon_{\mathrm{inc}} \cdot \rho^{[k]}, & \text { if }\left\|r^{[k+1]}\right\|_{2}>\mu\left\|s^{[k+1]}\right\|_{2} \\ \rho^{[k]} / \varepsilon_{\mathrm{dec}}, & \text { if }\left\|s^{[k+1]}\right\|_{2}>\mu\left\|r^{[k+1]}\right\|_{2} \\ \rho^{[k]}, & \text { otherwise, }\end{cases}
$$

where $\mu>0, \varepsilon_{\text {inc }}>1$ and $\varepsilon_{\mathrm{dec}}>1$ are the tunable parameters. $r^{[k+1]}:=x^{[k+1]}-z^{[k+1]}$ and $s^{[k+1]}:=-\rho^{[k]}\left(z^{[k+1]}-z^{[k]}\right)$ are referred to the primal residual and dual residual at step $k+1$, respectively.

The convergency of the ADMM algorithm can be judged by the primal residual $r$ and dual residual $s$. The iteration will be stopped when $\left\|r^{[k]}\right\|_{2}$ and $\left\|s^{[k]}\right\|_{2}$ satisfy,

$$
\left\|r^{[k]}\right\|_{2}<\epsilon^{\text {pri }} \text { and }\left\|s^{[k]}\right\|_{2}<\epsilon^{\text {dual }} .
$$


TABLE I

Overview of Voltage/Reactive Power Control Strategies for COMParison.

\begin{tabular}{|c|c|c|}
\hline No. & Strategy & Description \\
\hline S1 & Droop control & $\begin{array}{l}\text { The MV side bus of the transformer is selected as the pilot bus of the wind farm network. The } \\
\text { required reactive power is calculated based on the voltage at the pilot bus using droop-based } \\
\text { control strategy. All WTs hold fair reactive power sharing (proportional distribution). }\end{array}$ \\
\hline S2 & PI control & $\begin{array}{l}\text { The required reactive power is calculated based on the voltage at the pilot bus using a PI controller } \\
\text { and then proportionally dispatched to each WT. }\end{array}$ \\
\hline S3 & Centralized MPC & $\begin{array}{l}\text { In the centralized MPC scheme, the mathematical description of the optimal control problem is } \\
\text { the same as the proposed DCVCS but it is solved in a centralized manner. (to validate of the } \\
\text { proposed DCVCS) }\end{array}$ \\
\hline S4 & Loss minimization control & The LMC is also developed based on the sensitivity with the aim of minimizing grid loss. \\
\hline S5 & Conventional optimal control & $\begin{array}{l}\text { For the OPT, the control objectives are same as the MPC scheme, however, it is implemented } \\
\text { based on the one-step optimization without any prediction. }\end{array}$ \\
\hline
\end{tabular}

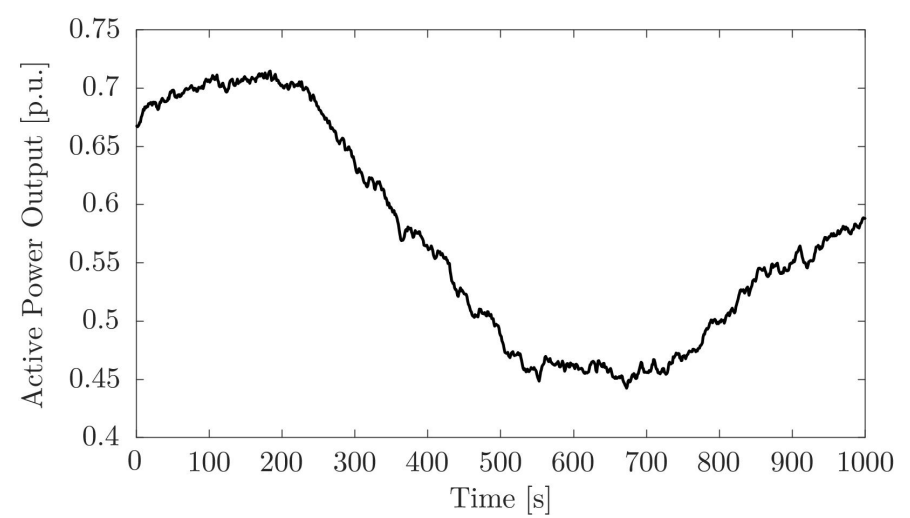

Fig. 5. Active power output of the wind farm.

\section{Case Study Setup}

In the case study, an offshore wind farm with $64 \times 6.25 \mathrm{MW}$ WTs, totally $400 \mathrm{MW}$, is used for the case study to verify the proposed DCVCS. The wind farm is connected to the onshore AC grid through a $\pm 150 \mathrm{kV}$ VSC-HVdc transmission system, of which the topology has been illustrated in Fig. 1. The WTs are connected with $33 \mathrm{kV}$ cables. There are eight WTs at each feeder, referred to as a string with a distance of $1.5 \mathrm{~km}$ between two WTs. The wind field modeling considering turbulences and wake effects for the wind farm was generated from the SimWindFarm Toolbox [25].

The control period $T_{c}$ and prediction horizon $H_{p}$ are designed as $1 \mathrm{~s}$ and $5 \mathrm{~s}$, respectively. In the ADMM framework, the parameters for $\rho$-updating are set as $\varepsilon_{\mathrm{inc}}=\varepsilon_{\mathrm{dec}}=5$ and $\mu=10$. The tolerances $\epsilon^{\text {pri }}$ and $\epsilon^{\text {dual }}$ are both set as $10^{-10}$. The simulation time is $1000 \mathrm{~s}$. For the active power control, the wind farm is assumed to operate in the maximum power point tracking (MPPT) mode. The total power output of the wind farm is shown in the Fig. 5.

The proposed decentralized control scheme is compared with the conventional control strategies such as PI control [7] and droop control [8], as well as the optimization-based control strategies including the centralized MPC (with the same optimization model), loss minimization control (LMC) and conventional optimal control (OPT) [10], which are described in Table I.

To quantify the overall voltage control performance of different control strategies, a dynamic voltage performance index (VPI) is defined as,

$$
\mathrm{VPI}=\sqrt{\sum_{i=1}^{N}\left(V_{i}-V_{\mathrm{ref}}\right)^{2}} .
$$

It can provide a comparison of control performances with different control schemes.

\section{Simulation Results}

In this section, the decentralized MPC is compared with the centralized MPC (S3) to validate the optimality of the decentralized solution. Besides, the voltage control performance of the MPC is compared with that with other existing control schemes (S1, S2, S4 and S5).

\section{A. Centralized vs. Decentralized MPC}

Figs. 6 and 7 show the voltage of VSC-controlled bus and terminal voltage of WT- 08 and Fig. 8 represents the whole voltage profile across the network. Fig. 9 shows the reactive power set-point of WT-04. And as shown in the zoomed part in Fig. 7, the difference of $V_{W_{8}}$ between the centralized and decentralized control is less than 0.0001 p.u., implying that the centralized and decentralized control schemes have the similar control performances. As known, in the dynamic simulation, the differences of the control performance can be accumulated over time, indicating that the decentralized and centralized control should have the same (high accuracy) control commands at each control point if the same control performances are kept over time. This can be further verified by Fig. 9, in which reactive power set-point of the WT can be considered identical at each control point. Thus, it can be concluded that the proposed ADMM-based decentralized framework can guarantee the optimality of the primal problem. 


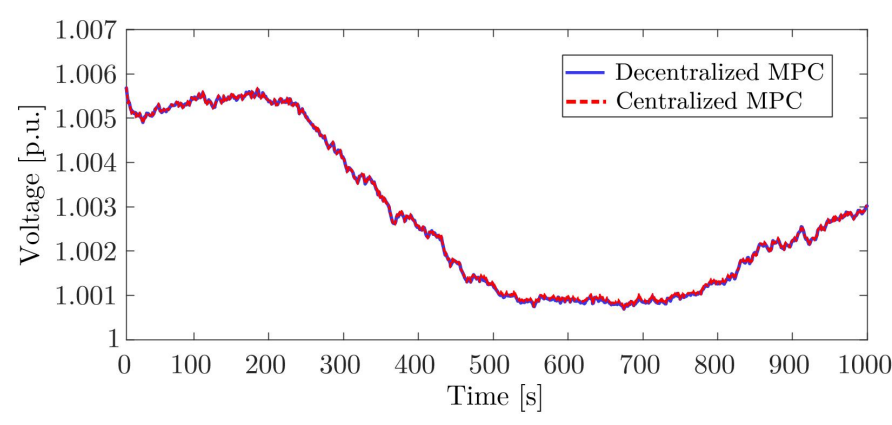

Fig. 6. Voltage of WFVSC-controlled bus (imposed by the VSC-HVdc).

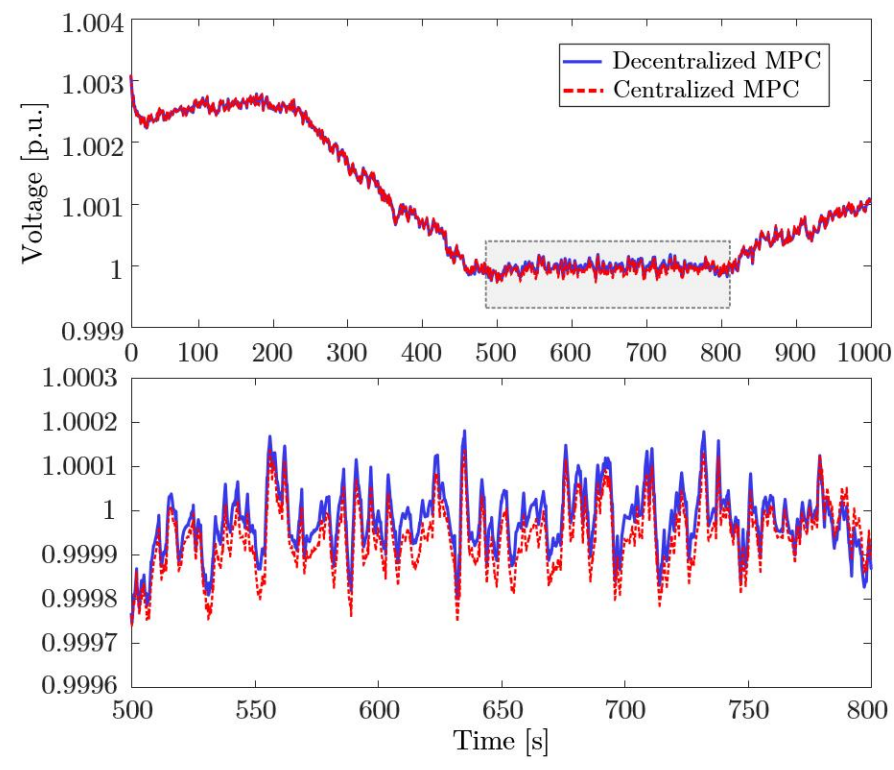

Fig. 7. Terminal voltage of WT- 08 .

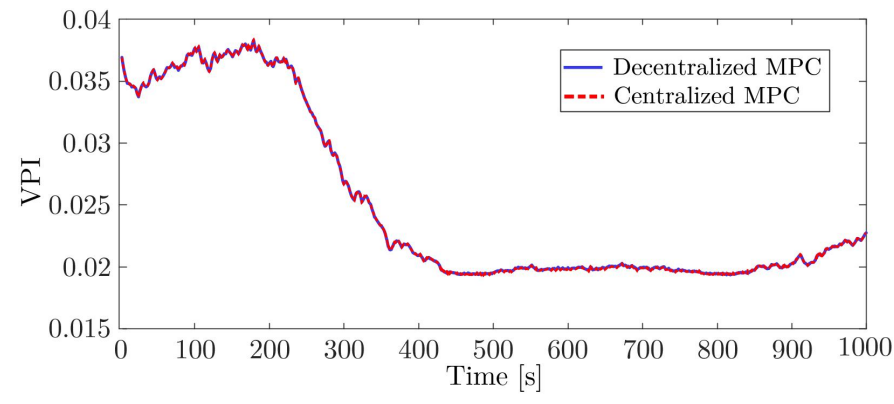

Fig. 8. Overall voltage control performance.

\section{B. MPC vs. Droop Control, PI control, LMC and OPT}

The proposed decentralized MPC scheme is compared with other existing methods. The simulation results are presented in Figs. 10-14.

1) PI control and droop control: As can be seen from Fig. 11 , the PI control and droop control can effectively regulate the pilot bus voltage within the feasible range. Especially for the PI control, the pilot bus voltage can be accurately controlled around 1.0 p.u.. However, the voltage at the WT buses (see Fig. 12, WT-08) could reach 1.02-1.04 p.u., since these two control schemes are established only based on the voltage at the

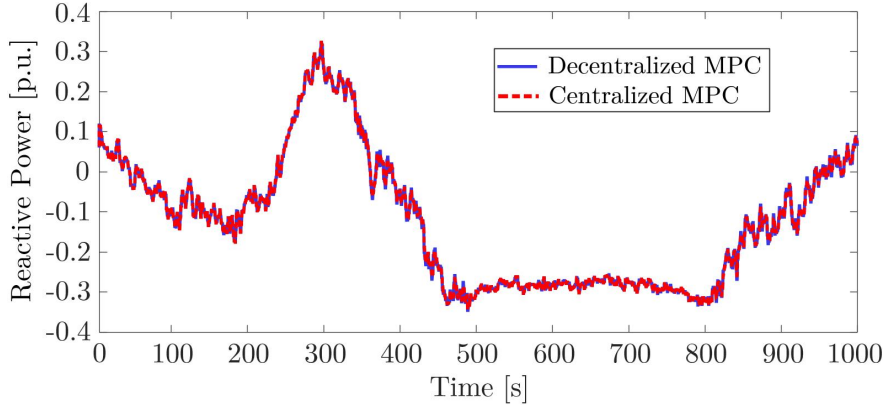

Fig. 9. Reactive power output of WT-04.

pilot bus. Comparably, the MPC scheme can more effectively regulate the voltage at the WT buses within the range of 0.99-1.01 p.u.. As shown in Fig. 13, the quantified overall performance index VPI gives a clear comparison, which shows the MPC scheme is much better than the PI control and droop control.

2) OPT: Figs. 10 and 12 illustrate the voltage at the VSCcontrolled bus and terminal voltage of WT- 08 . As can be seen, both of the OPT and MPC can regulate the network voltages within the feasible range. However, the MPC can better mitigate the voltage fluctuations caused by the active power output variations of WTs, due to the prediction mechanism and multi-step optimization. It can be directly observed from Fig. 13, the overall voltage control performance with the MPC is better than that with the OPT.

3) $L M C$ : The LMC is designed with the aim of minimizing the grid losses. As can be seen from Fig. 14, the LMC can more effectively reduce the grid losses compared with the MPC. The average of losses over the time (with the sampling time of $1 \mathrm{~s}$ ) is $9.016 \mathrm{MW}$ for the MPC and $8.2093 \mathrm{MW}$ for the LMC. However, the voltage profile across the network is much higher than the nominal value. The terminal voltages of WT can reach 1.05 p.u., indicating a high risk of being tripped in case of a large disturbance. From Fig. 13, it can be observed that the voltage deviations with the MPC is much smaller than that with the LMC implying the much better voltage control performance.

\section{DISCUSSION}

The advantages of the proposed decentralized voltage control against the centralized voltage control are summarized as follows.

\section{A. Scalability}

Firstly, as mentioned before, the large-scale constrained optimization problem is divided into an unconstrained QP problem and multiple parallel small-scale constrained optimization problems. The central agent is just required to solve an unconstrained QP problem which can be solved by the simple matrix calculation and the small-scale constrained optimization problems are locally solved in parallel. Compared with the centralized control, the proposed decentralized control significantly reduces the computation burden of the central controller and the computation efficiency will be slightly affected by the increasing number of WTs. 

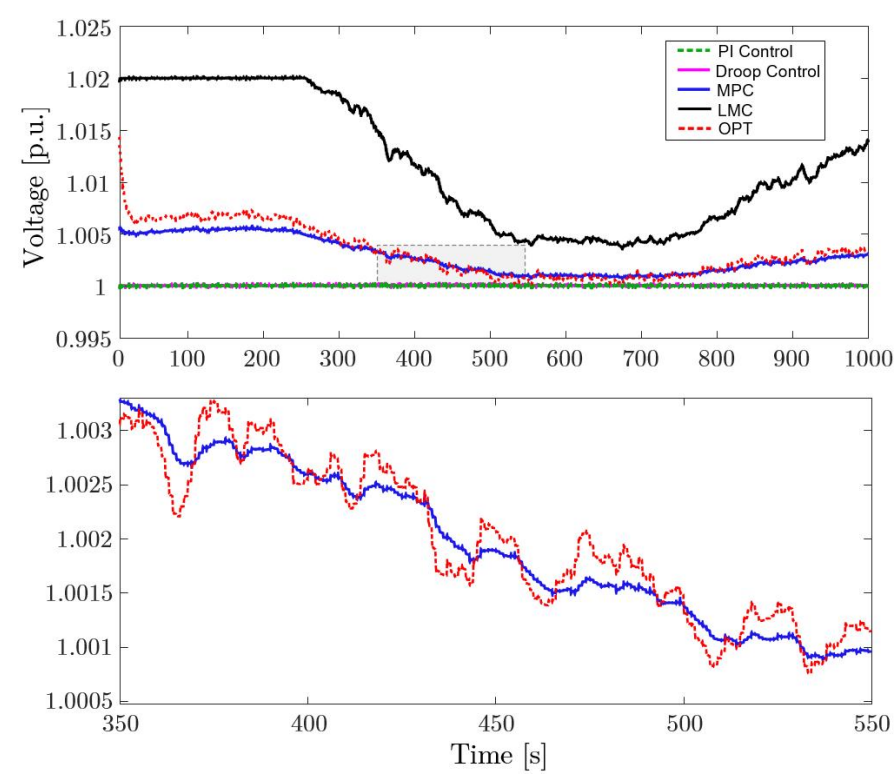

Fig. 10. Voltage of WFVSC-controlled bus (imposed by the VSC-HVdc).

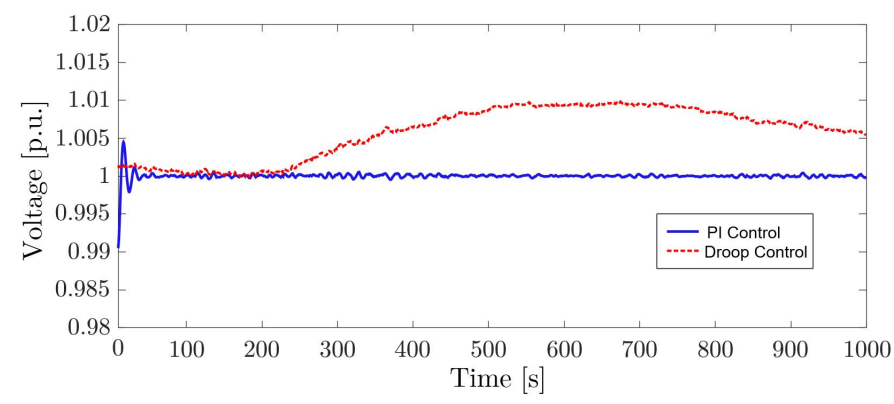

Fig. 11. Voltage of pilot bus.

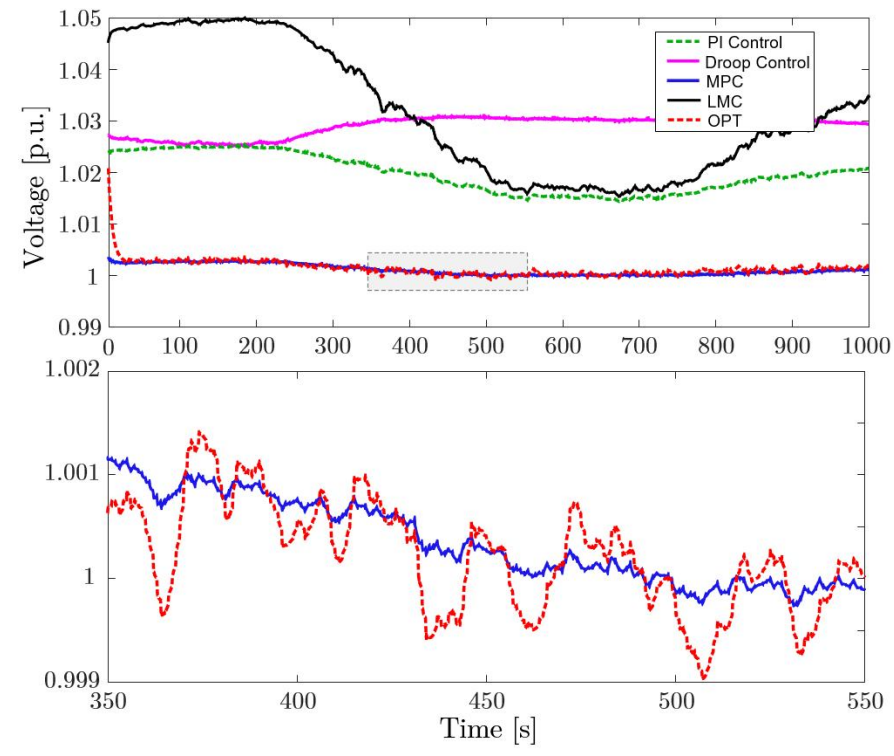

Fig. 12. Terminal voltage of WT- 08 .

\section{B. Cyber Security}

With higher penetration of wind generation, as well as the advanced cyber components and networks, cyber attacks
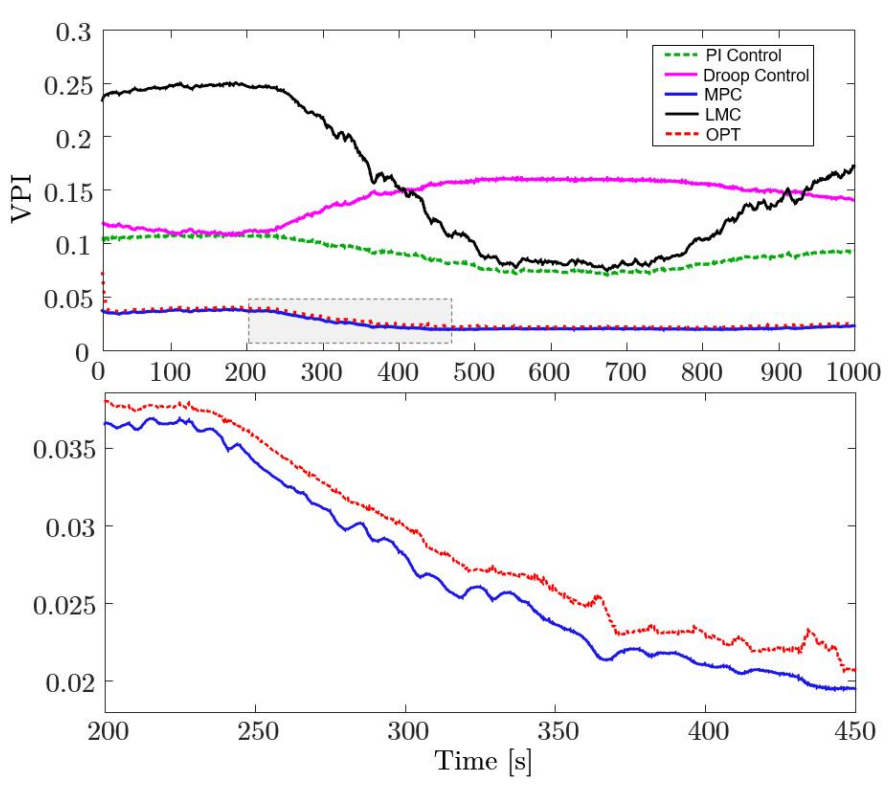

Fig. 13. Overall voltage control performance.

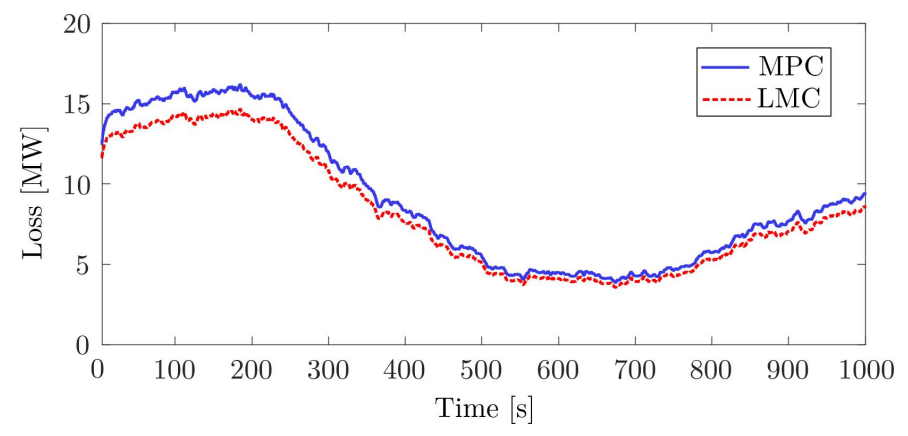

Fig. 14. Grid loss comparison between the MPC and LMC.

against the wind farms will become a non-negligible factor which might be a potential threat for power system operation [27]. In the conventional centralized control schemes, the central wind farm controller directly acquires the measured power outputs of WTs from each WT, solves the optimization problem and then sends the reactive power set-points to each WT. For the decentralized control, the central agent and local agent exchange the updating variables in the ADMM, $x, z, \gamma$ and $\rho$, instead of explicit reactive power measurements and setpoints. From the perspective of cyber and information security, the decentralized manner can better prevent the operating information of the wind farms from being directly stolen by the hackers, which is one of the common attack scenarios for wind farms [27].

\section{Flexibility and Robustness}

The existence of local agents provides the possibility of designing local control strategies as the back-up control in the local agents such as droop-based control without any communication which has faster response time than the centralized optimization-based control strategies. Once the central agents fails to work or are under emergence conditions, the local agents can fast regulate the voltages in some degree 
even though it might not be the global optimal solution, implying better flexibility and robustness than the centralized ones which fully depend on the central controller.

\section{CONCLUSION}

The paper proposes a decentralized coordinated voltage control scheme for VSC-HVdc connected wind farms. Firstly, the MPC-based voltage control problem of wind farms is formulated as an optimization model. Then, it is solved in a decentralized manner using the ADMM algorithm. The simulation results show that the decentralized voltage control has the same control performance as the centralized one, without loss of optimality of the primal problem. Compared with other existing control methods such as the PI control, droop control, LMC and OPT, the proposed MPC scheme can better regulate the voltages with the smaller fluctuations and deviations. Owing to its potential advantages on the scalability, cyber security, flexibility, and robustness, the decentralized control is preferred to the centralized control for the largescale OWFs or OWF clusters with hundreds or even thousands of WTs in the near future.

The unified voltage profile is expected in the wind farm in this study. However, for a large-scale wind farm with long-distance collector feeders, the optimal voltage gradient along the feeders will be further investigated in our future work. Moreover, since the optimality of the decentralized framework has been validated, it can be extended to deal with other optimal control problems with the similar structure of mathematical models.

\section{REFERENCES}

[1] Z. Miao, L. Fan, D. Osborn, and S. Yuvarajan, "Control of DFIG-based wind generation to improve inter-area oscillation damping," IEEE Trans. Energy Convers., vol. 24, no. 2, pp. 415-422, Jun. 2009.

[2] K. Reichardt, K. S. Rogge, S. O. Negro, "Unpacking policy processes for addressing systemic problems in technological innovation systems: The case of offshore wind in Germany," Renew. Sustain. Energy Rev., vol. 80, pp. 1217-1226, 2017.

[3] M. Bilgili, A. Yasar, and E. Simsek, "Offshore wind power development in Europe and its comparison with onshore counterpart," Renew. Sustain. Energy Rev., vol. 15, pp. 905-915, 2011.

[4] R. Zeng, L. Xu, L. Yao, S. J. Finney, and Y. Wang, "Hybrid HVDC for integrating wind farms with special consideration on commutation failure," IEEE Trans. Power Del., vol. 31, no. 2, pp. 789-797, Apr. 2016.

[5] P. Hou, W. Hu, M Soltani, C. Chen, B. Zhang, Z. Chen, "Offshore wind farm layout design considering optimized power dispatch strategy," IEEE Trans. Sustain. Energy, vol. 8, no. 2, pp. 638-637, Apr. 2017.

[6] X. Wang, W. Gao, A. Scholbrock, E.Muljadi, V. Gevorgian, V. Gevorgian, J. Wang, W. Yan H. Zhang, "Evaluation of different inertial control methods for variable-speed wind turbines simulated by fatigue, aerodynamic, structures and turbulence (FAST)," IET Renew. Power Gener, vol. 11, no. 12, pp. 1534-1544, 2017.

[7] B. Karthikeya and R. Schutt, "Overview of wind park control strategies," IEEE Trans. Sustain. Energy, vol. 5, no. 2, pp. 416-422, Apr. 2014.

[8] J. Martínez, P. C. Kjær, P. Rodriguez, and R. Teodorescu, "Comparison of two voltage control strategies for a wind power plant," in Proc. IEEE/PES Power Syst. Conf. Expo. (PSCE), 2011, pp. 1-9.

[9] B. Zhang, P. Hou, W. Hu, M. Soltani, C. Chen, and Z. Chen, "A Reactive Power Dispatch Strategy With Loss Minimization for a DFIG-Based Wind Farm," IEEE Trans. on Sustain. Energy, vol. 7, no. 3, pp. 914-923, Jul. 2016.

[10] Q. Guo, H. Sun, B. Wang, B. Zhang, W. Wu and L. Tang, "Hierarchical automatic voltage control for integration of large-scale wind power: Design and implementation," Electr. Power Syst. Res., vol. 120, pp. 234$241,2015$.
[11] H. Zhao, Q. Wu, Q. Guo, H. Sun, S. Huang, and Y. Xue, "Coordinated voltage control of a wind farm based on model predictive control", IEEE Trans. Sustain. Energy, vol. 7, no. 4, pp. 1440-1451, Oct. 2016.

[12] H. Zhao, Q. Wu, J. Wang, Z. Liu, and M. Shahidepour, and Y. Xue, "Combined active and reactive power control of wind farms based on model predictive control," IEEE Trans. Energy Convers., vol. 32, no. 3 , pp. 1177-1187, Sep. 2017.

[13] M. Martinez-Rojas, A. Sumper, O. Gomis-Bellmunt, and A. SudriàAndreu, "Reactive power dispatch in wind farms using particle swarm optimization technique and feasible solutions search," Applied energy, vol. 88 , no. 12 , pp. 4678-4686, 2011.

[14] M. Montilla-DJesus, D. Santos-Martin, S. Arnaltes, and E. D. Castronuovo,"Optimal reactive power allocation in an offshore wind farms with LCC-HVdc link connection," Renew. energy, vol. 40, no. 1, pp. 157-166.

[15] K. Schönleber, S. Ratés-Palau, M. De-Prada-Gil, and O. GomisBellmunt, "Reactive power optimization in HVDC-connected wind power plants considering wake effects," 14th Wind Integr. Work, Energynautics GmbH, Brussels, 2015.

[16] K. Schönleber, C. Collados, R. T. Pinto, S. Ratés-Palau, and O. Gomis-Bellmunt,"Optimization-based reactive power control in HVDCconnected wind power plants," Renew. Energy, vol. 109, pp. 500-509, 2017.

[17] J. N. Sakamuri, Z. H. Rather, J. Rime, M. Altin, Ö. Goksu, and N. A. Cutululis, "Coordinated voltage control in offshore HVDC connected cluster of wind power plants," IEEE Trans. Sustain. Energy, vol. 7, no. 4, pp. 1592-1601, Oct. 2016.

[18] K. Schönleber, S. Ratés-Palau, O. Gomis-Bellmunt, "Analysis of reactive power strategies in HVDC-connected wind power plant clusters," Wind Energy, vol. 20, pp. 1971-1982, 2017.

[19] Y. Guo, H. Gao, Q. Wu, H. Zhao, and J. Østergaard, "Coordinated voltage control scheme for VSC-HVDC connected wind power plants," IET Renew. Power Gener, vol. 12, no. 2, pp. 198-206, 2018.

[20] Y. Guo, H. Gao, Q. Wu, H. Zhao, J. Østergaard, and M. Shahidehpour, "Enhanced voltage control of VSC-HVDC connected offshore wind farms based on Model Predictive Control," IEEE Trans. Sustain. Energy, vol. 9, no. 1, pp. 474-487, Jan. 2018.

[21] K. Christakou, J. LeBoudec, M. Paolone, and D.-C. Tomozei, "Efficient computation of sensitivity coefficients of node voltages and line currents in unbalanced radial electrical distribution networks," IEEE Trans. Smart Grid, vol. 4, no. 2, pp. 741-750, Jun. 2013.

[22] S. K. Chaudhary, Control and Protection of Wind Power Plants with VSC-HVDC Connection, Ph.D. thesis, Dept. of Electr. Eng. Tech., Aalborg University, Aalborg, Denmark, 2011.

[23] E. V. Larsen and A. S. Achilles, "System and method for voltage control of wind generators," US Patent App. 14/018482, Sep. 52013.

[24] A. Maknouninejad and Z. Qu, "Realizing unified microgrid voltage profile and loss minimization: A cooperative distributed optimization and control approach," IEEE Trans. Smart Grid, vol. 5, no. 4, pp. 1621-1630, Apr. 2014.

[25] J. D. Grunnet M. Soctani, T. Knudsen, M. Kragelund, and T. Bak, "Aeolus toolbox for dynamic wind farm model, simulation and control," in Proc. Eur. Wind Energy Conf. Exihib., Warsaw, Poland, Apr. 2010, pp. $1-10$.

[26] S. Boyd, N. Parikh, E. Chu, B. Peleato and J. Eckstein, "Distributed optimization and statistical learning via the alternating direction method of multipliers," Found. Trends Mach. Learn, vol. 3, no. 1, pp. 1-122, Jan. 2011.

[27] Y. Zhang, Y. Xiang, and L. Wang, "Power system reliability assessment incorporating cyber atacks against wind farm energy management systems," IEEE Trans. Smart Grid, vol. 8, no. 5, pp. 2343-2357, 2016.

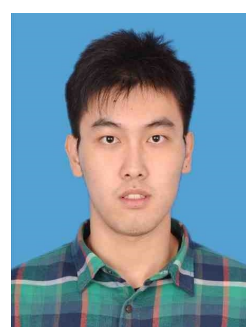

Yifei Guo received the B.E. degree in electrical engineering from Shandong University, Jinan, China, in 2014. Currently, he is pursuing the Ph.D. degree in electrical engineering at Shandong University. During 2017-2018, he is a visiting student in the Department of Electrical Engineering, Technical University of Denmark, Lyngby, Denmark. His research interests include voltage control, wind power integration, and power system protection. 


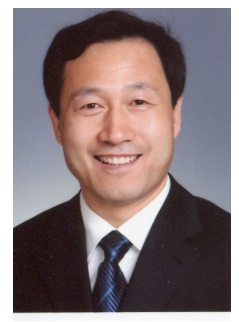

Houlei Gao was born in Shandong, China, in 1963. He received the B.Sc. and M.Sc. degrees in electrical power engineering from Shandong University, Jinan, China, in 1983 and 1988, respectively, and the Ph.D. degree from Tianjin University, Tianjin, China, in 1997. From 2004 to 2005, he was with the School of Electrical and Electronic Engineering, Queens University Belfast, Belfast, U.K. He is a Professor at the School of Electrical Engineering, Shandong University. His research interests include power syseration, and digital substation. em protection, feeder automation, distributed gen-

Hao Xing is an Assistant Professor with the Hangzhou Dianzi University, Hangzhou, China.

Qiuwei Wu is a Professor with the School of Electrical Engineering, Shandong University, Jinan, China.

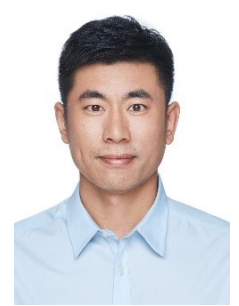

Zhongwei Lin received the B.E. and M.E. degree from Shandong University of Science and Technology in 2004 and 2007, respectively, and the Ph.D. degree from Beijing University of Aeronautics and Astronautics in 2011. He is now an Associate Professor with the School of Control and Computer Engineering at North China Electric Power University, Beijing, China. His research interests include stochastic control, nonlinear control, wind turbine modeling and control. 